\title{
EXPERIMENTAL AND NUMERICAL INVESTIGATIONS OF CONCRETE BEHAVIOUR AT MESO-LEVEL DURING QUASI-STATIC SPLITTING TENSION
}

\author{
J. SUCHORZEWSKI ${ }^{1}$ AND J. TEJCHMAN' \\ ${ }^{1}$ Gdańsk University of Technology \\ Faculty of Civil and Environmental Engineering \\ 80-233 Gdańsk-Wrzeszcz, Narutowicza 11/12 \\ jan.suchorzewski@pg.gda.pl,tejchmk@pg.gda.pl
}

Key words: Concrete, Splitting Tensile Test, DEM, Fracture, Loading Strip, Meso-scale.

\begin{abstract}
The paper describes experimental and numerical results of quasi-static splitting tensile tests on concrete specimens at meso-scale level. The loading strip was made of plywood or steel. Fracture in concrete was detected at the aggregate level by means of three nondestructive methods: 3D x-ray micro-computed tomography, 2D scanning electron microscope and manual 2D digital microscope. The discrete element method was used to directly simulate experiments at the meso-scale. Concrete was modelled as a random heterogeneous 4-phase material composed of aggregate particles, cement matrix, interfacial transitional zones and macro-voids based on micro-tomographic images. Two-dimensional calculations with real concrete microstructure were carried out. A satisfactory agreement between numerical and experimental results was achieved. The evolution of contact normal forces, coordination number, broken contacts, grain rotations and crack displacements was also investigated. In addition, each energy component was calculated and analyzed at a different stress-displacement stage.
\end{abstract}

\section{INTRODUCTION}

The splitting tensile tests (also known as the Brazilian tests) are the most popular laboratory tests on concrete to determine its uniaxial tensile strength due to their loading and specimen shape simplicity. This test consists of applying a distributed compressive force along the length of a concrete cylinder, which induces a primarily tensile stress perpendicular to the loading plane of the specimen's cross-section with a sharp compressive stress near the points of load application. The tests are however sensitive to boundary conditions related to the width, shape and stiffness of a loading strip. The effect of boundary conditions on the initial global stiffness and post-peak behaviour of concrete specimens (strength-displacement curve, fracture) has not been investigated yet.

The fracture process in the splitting test with standard loading strips consists of two main stages: (1) a main macro-crack formation in the central vertical zone and (2) secondary cracks connecting the main vertical crack with edges of loading plates. In addition, the test outcomes 
are subjected to a size effect, expressed by a decrease of both the nominal strength and ductility with increasing specimen diameter [1].

Our experimental and theoretical research works are aimed at understanding the concrete behaviour at the meso-scale during different failure modes in quasi-static splitting tension, depending upon the specimen diameter. Based on preliminary experiments, a quasi-brittle concrete behaviour took place with small specimen diameters $(D=5 \mathrm{~cm})$. For larger diameters $(D=10 \mathrm{~cm})$, a brittle concrete behaviour occurred. Finally, for large diameters $(D \geq 15 \mathrm{~cm})$, a snap-back instability was observed (which is described by a positive slope in a stress-strain softening branch). In this paper concrete cylinders with the diameter of $0.15 \mathrm{~m}$ were experimentally and theoretically investigated. During laboratory tests, a snap-back instability occurred. The vertical load was transferred to concrete specimens through a steel cylinder (in the form of a line contact) or a plywood board (in the form of a surface contact). Fracture was monitored using a high resolution and non-destructive technique in the form of the 3D x-ray micro-computed tomography (using Skyscan 1173 [2]). In addition, the 2D scanning electron microscope (SEM) Hitachi TM3030 and manual 2D digital microscope 'Scalar' were used. The discrete element method (DEM) was applied to describe a non-linear global response of concrete during splitting tension. In this method concrete was considered at the meso-scale as 4-phase material (aggregate, cement matrix, macro-voids and interfacial transitional zones (ITZs)). The geometry of concrete micro-structure at the meso-level was incorporated into DEM from real concrete specimens by means of micro-tomography images. The model was successfully applied to concrete under bending and compression [3], [4]. These calculations showed that it was of importance to take into account the shape and position of aggregate particles and strength and number of ITZs for a realistic reproduction of concrete fracture. ITZs due to a porous structure acted as attractors for cracks and thus influenced the strength and brittleness of concrete. DEM was also used to the fracture description in concrete by other researchers. The splitting tensile tests for quasi-brittle materials were mainly simulated within continuum mechanics.

The main goal of the paper is threefold: 1) to check the capability of DEM for simulating quasi-static splitting tension in concrete specimens at the meso-level by taking a snap-back instability into account, 2) to check the effect of different boundary conditions on the strength and fracture (expressed by the different loading and support strip type) and 3) to investigate in detail the concrete behaviour during quasi-static splitting tension at the meso-scale level. The numerical outcomes were directly compared with the experimental results with respect to the measured stress-strain curves and observed crack patterns based on $3 \mathrm{D} \mu \mathrm{CT}$-images. The evolution of contact forces, broken contacts, internal energies and crack displacements at the aggregate level were also investigated by DEM. The innovative point is to the use of a 4phase concrete mesoscopic model for fracture investigations based on the real internal concrete structure based on $\mu \mathrm{CT}$. In the future the DEM calculations may replace laboratory tests to investigate the influence of the concrete meso-structure on its global behaviour. 


\section{EXPERIMENTS}

\subsection{Strength}

The splitting tensile experiments were performed in the static loading machine ZWICK Roaller Z400 (Fig.1). The machine was equipped with a crack opening extensometer (Sandner EXR10-2x) within the measurement range of $\pm 2 \mathrm{~mm}$ with the maximum error of $2 \%$. The extensometer base was equal to $40 \mathrm{~mm}$. The extensometer was located at the mid-height of the concrete specimen and glued to the specimen front side (Fig.1). The quasi-static tests were performed under the CMOD-control (CMOD - crack mouth opening displacement) with the displacement rate of $0.00001\left(1 \times 10^{-5}\right) \mathrm{mm} / \mathrm{s}$. Two types of loading/supporting strips were used: deformable plywood boards with the thickness of $t=3 \mathrm{~mm}$ and width of $b=25 \mathrm{~mm}$ (Fig. 1b) (as recommended by ASTM [5]) and rigid steel loading/supporting cylinders with the diameter of 20 mm (Fig. 1a). Thus the vertical load was respectively transferred to specimen through a surface or line.

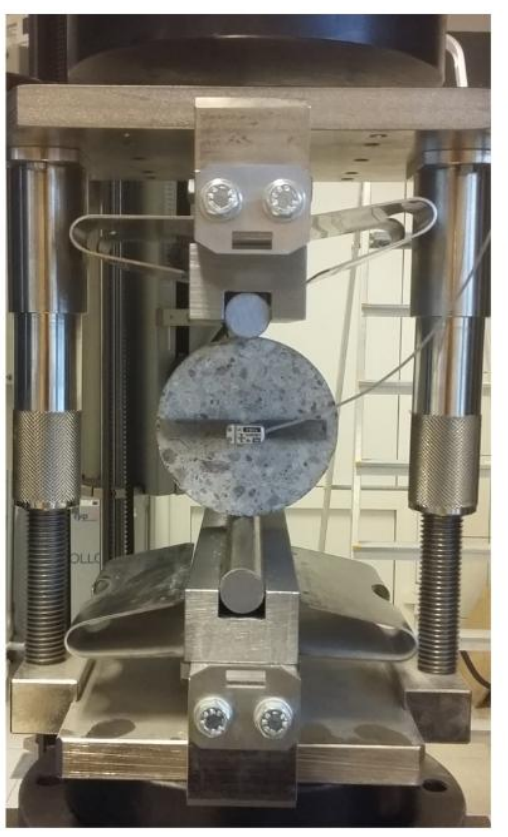

a)

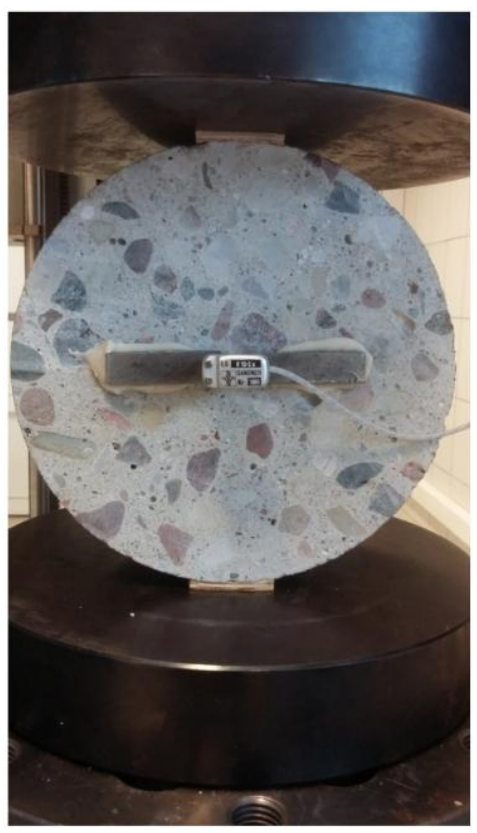

b)

Figure 1: View on loading machine Zwick Z400 with cylindrical concrete specimen of diameter $D=150 \mathrm{~mm}$ and length $L=60 \mathrm{~mm}$ for quasi-static splitting tensile tests: a) specimen loaded through steel cylinder and b) specimen loaded through plywood board

The minimum aggregate diameter was $d_{a}{ }^{\min }=2 \mathrm{~mm}$, maximum aggregate diameter was $d_{a}{ }^{\text {max }}=12 \mathrm{~mm}$ and mean aggregate diameter $d^{a}{ }_{50}=5 \mathrm{~mm}$. The volumetric aggregate sieve content was $31.2 \%$ (diameter $2-8 \mathrm{~mm}$ ), and $16.6 \%$ (diameter $8-12 \mathrm{~mm}$ ). Thus, the aggregate volumetric content was $47.8 \%$. The total particle volumetric content (sand and aggregate) in concrete was $75 \%$. The concrete specimens with the diameter of $D=150 \mathrm{~mm}$ were used. The minimum specimen length was assumed $L=60 \mathrm{~mm}\left(=5 \times d_{a}{ }^{\max }\right)$. The mean standard compressive strength of 
concrete tested on 3 cubic specimens $150 \times 150 \times 150 \mathrm{~mm}^{3}$ was equal to $f_{c, c u b e}=49.6 \mathrm{MPa}$ and mean standard modulus of elasticity tested on 3 cylindrical specimens $D=150 \mathrm{~mm}$ and length $L=300 \mathrm{~mm}$ was $E_{c}=33.1 \mathrm{GPa}$.

Several splitting tests on concrete were carried out. Figure $2 \mathrm{~A}$ presents the representative experimental stress versus CMOD curves for concrete specimens using two types of the loading/supporting contact along the specimens (steel cylinder and plywood board). The CMOD evolution during loading was perfectly linear in time. The splitting tensile strength, calculated as $\sigma=2 P_{\max } / \pi D L\left(P_{\max }\right.$ - the maximum vertical piston force), varied between $3.26 \mathrm{MPa}$ $\left(P_{\max }=47.2 \mathrm{kN}, v=0.45 \mathrm{~mm}, \mathrm{CMOD}=18 \mu \mathrm{m}\right)$ and $\sigma=3.64 \mathrm{MPa}\left(P_{\max }=51.2 \mathrm{kN}, v=1.15 \mathrm{~mm}\right.$, $\mathrm{CMOD}=22 \mu \mathrm{m}$ ) for the steel loading/supporting cylinders (line contacts) and plywood loading/supporting boards (surface contacts), respectively. Thus, the splitting tensile strength was higher by about $10 \%$ for the plywood board. Looking at the curve of the stress $\sigma$ versus CMOD (Fig.2A), initially concrete elastically behaved up to $70 \%$ of the maximum tensile stress $\sigma$ and later slightly non-linearly up to the peak load. After pronounced material softening occurred up to the residual state to failure. The residual stress was reached for $\sigma=2.3 \mathrm{MPa}$ (plywood boards) and $\sigma=1.9 \mathrm{MPa}$ (steel cylinder) for $\mathrm{CMOD}=110 \mu \mathrm{m}$. When considering the experimental stressvertical piston displacement diagram $\sigma=f(v)$ (Fig.2B), a clear snap-back instability occurred for $v=1.15 \mathrm{~mm}$ (plywood loading board) and $v=0.45 \mathrm{~mm}$ (steel loading cylinder), expressed by a simultaneous reduction of the stress and displacement. Shortly before the test end, the displacement $v$ slightly increased due to the specimen defragmentation.

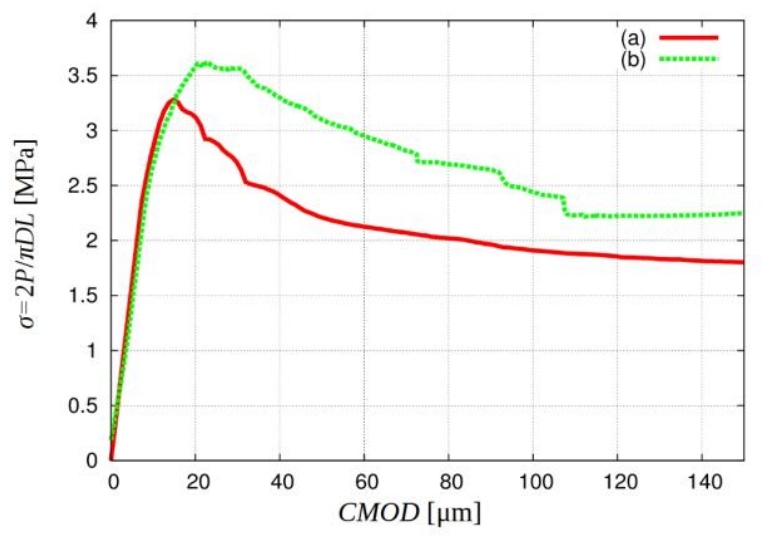

A)

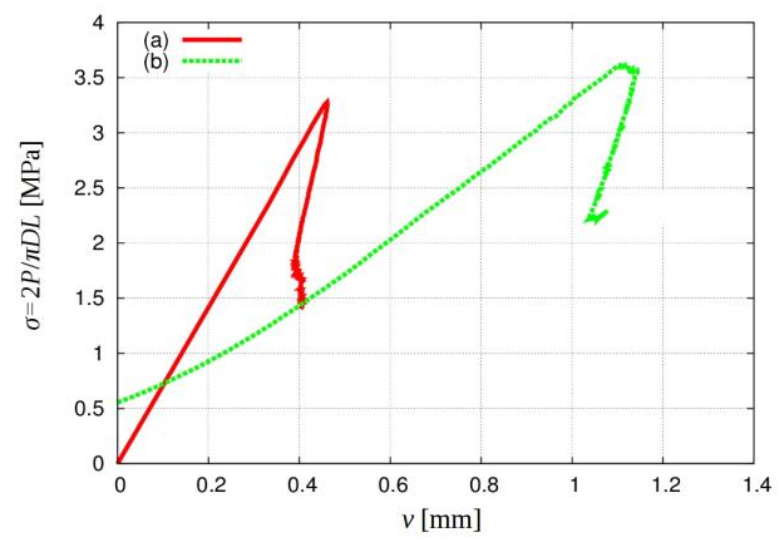

B)

Figure 2: Experimental curves for concrete specimens with diameter $D=0.15 \mathrm{~m}$ ): A) splitting tensile stress $\sigma=2 P(\pi D L)$ versus CMOD and B) splitting tensile stress $\sigma$ versus top vertical displacement $v$ for 2 different loading systems a) steel cylinder and b) plywood board ( $P$ - vertical force, $L$ - specimen length)

In all tests the main vertical macro-crack first occurred at the mid-height of the specimen in the central vertical zone (Fig.3). Then it propagated towards both the specimen top and bottom. Later depending upon the loading and support strip type it reached the top and the bottom of the specimen (Fig.3a) or branched to form a wedge directly under the plywood board (Fig.3b). Finally at failure, the concrete specimen was divided into two halves. 


\subsection{Concrete porosity}

In order to measure more precisely the concrete porosity, a smaller concrete specimen ( $D=50 \mathrm{~mm}$ and $L=60 \mathrm{~mm}$ ) was cut out from the same concrete block and scanned by means of the 3D x-ray micro-tomograph Skyscan 1173. The x-ray micro-tomography (called micro-CT or $\mu \mathrm{CT})$ is a 3D imaging technique which uses $\mathrm{x}$-rays to create cross-sections of a physical object that is used to recreate a virtual model (3D model) without destroying the original object. Our x-ray micro-tomograph represents a new generation in high-resolution desktop X-ray microtomography systems [2]. The scans were completed to ten times faster with the same resolution and image quality as compared to previous micro-CT with a fixed source-detector design. The scanner was equipped with the newly developed $130 \mathrm{keV}$ microfocus $\mathrm{x}$-ray source with a very stable focal spot position and flat panel sensor of a large format (5 Mpx) with a special protection by a lead-glass fibre-optic window. As compared to usual X-ray micro-tomographs, this scanner has two basic advantages: a) large specimens up to $150 \mathrm{~mm}$ in diameter may be scanned and b) specimens are scanned with a higher precision (2-3 microns). Note that a continuous investigation of the entire fracture process under deformation with our X-ray micro-tomograph has not been possible for technical reasons yet. The measured total volume of voids was $p=3.2 \%$ and the measured volume of voids with the equivalent diameter $d_{p}<1 \mathrm{~mm}$ was $p=1.6 \%$.

\subsection{Concrete fracture}

The macro-crack images by means of the 3D x-ray micro-tomograph Skyscan 1173 are depicted in Fig. 3 for the damaged specimen of Fig.3a after the test $(D=0.15 \mathrm{~m}, L=0.06 \mathrm{~m})$. In order to obtain a better accuracy in the X-ray micro-tomograph, the specimen width was diminished to $0.08 \mathrm{~m}$ after the test. The particular phases are shown in Fig.3A. The macro-crack was curved along the vertical and horizontal plane due to a random presence of aggregate grains (Figs.3B-3D). The micro-cracks always initiated in ITZs along aggregate particles since they were the weakest phase in concrete. When two interfacial cracks occurred around adjacent aggregates, a crack inside the cement matrix initiated to bridge the interfacial cracks so that a connected crack path was formed. Sometime it also crossed macro-voids and aggregate. On the specimen front side, the macro-crack crossed e.g. 3 aggregate particles. The crack branching also occurred.

In order to determine ITZs on the front surface of non-damaged specimens, the scanning electron microscope (SEM) HITACHI TM3030 with the maximum magnification factor 30 '000 was used. The small specimen $40 \times 40 \times 1.5 \mathrm{~mm}^{3}$ was used which was cut out from the initial concrete block. ITZs around particles were characterised by a porous structure as compared to the cement matrix (Fig.8). Their width varied between $10 \mu \mathrm{m}$ and $25 \mu \mathrm{m}$. They appeared around all aggregate grains $\left(d_{a} \geq 2 \mathrm{~mm}\right)$ and usually covered about $80-90 \%$ of the aggregate circumference that was probably caused by a formation of water lenses beneath aggregate grains during mixing. The width of ITZs was not connected with the aggregate diameter. Other experimental results showed that the width of ITZ reduced with decreasing aggregate roughness.

\section{DISCRETE ELEMENT METHOD MODEL FOR CONCRETE}

The 3D spherical discrete element model YADE takes advantage of the so-called soft-particle approach (i.e. the model allows for particle deformation which is modelled as an overlap of 
particles) [6]. A linear normal contact model under compression was used. Aggregate grains were modelled as clusters composed of spheres. The interaction force vector representing the action between two spherical discrete elements in contact was decomposed into a normal and tangential vector, respectively. The normal forces acting on spheres were modelled by an elastic law with cohesion. The normal and tangential forces were linked to the displacements through the normal stiffness $K_{n}$ and the tangential stiffness $K_{s}$. The stiffness parameters were computed with the aid of the modulus of elasticity of the grain contact $E_{c}$ and two neighbouring grain radii $R_{A}$ and $R_{B}$ (to determine the normal stiffness $K_{n}$ ) and with the aid of the modulus of elasticity $E_{c}$ and Poisson's ratio $v_{c}$ of the grain contact and two neighbouring grain radii $R_{A}$ and $R_{B}$ (to determine the tangential stiffness $K_{s}$ ), respectively [6].

The contact tangential forces $F_{s}$ and normal forces $F_{n}$ satisfied the cohesive-frictional MohrCoulomb equation using the inter-particle friction angle $\mu$ [6]. The normal force might be negative down to the minimum value of $F_{n}$ min if there was no a geometrical contact between elements. If this minimum normal force between spheres $F_{n}$ min was reached, the contact was broken. Moreover, if any contacts between grains re-appeared, cohesion between them was not taken into account. A crack was considered as open if cohesive forces between grains disappeared when a critical threshold was reached. A choice of a very simple linear elastic normal contact was intended to capture on average various contact possibilities in real concrete. One assumed that the cohesive force and tensile force were a function of the cohesive stress $C$ (maximum shear stress at pressure equal to zero), tensile normal stress $T$ and sphere radius $R$. To dissipate excessive kinetic energy in a discrete system, a simple local non-viscous damping scheme was adopted which assumed a change of forces by using the damping parameter.

Concrete was described in DEM computations as a four-phase material composed of aggregate, cement matrix interfacial transitional zones (ITZs) and macro-voids. The numerical analyses were performed on concrete specimens with the same aggregate location and shape as in experiment. In the first step, two-dimensional simulations were performed to significantly reduce the computation time. Thus the crack curvature along the specimen length was not taken into account. The 3D analyses significantly increase computation times but do not significantly affect the results except of a small reduction of material brittleness. The voids were modelled simply as empty regions with a real shape. In order to create the real aggregate shape in 2D calculations $\left(2 \mathrm{~mm} \leq d_{a} \leq 12 \mathrm{~mm}\right)$ based on images of the polished specimen surface and $\mu \mathrm{CT}$ scan, the clusters composed of spheres with the diameter of $d=1.0 \mathrm{~mm}$ connected to each other as rigid bodies were used. One aggregate particle, depending upon its diameter, included 5-500 spheres. Based on experiments all aggregate grains with the diameter in the range of $2 \mathrm{~mm}<d_{a<}$ $12 \mathrm{~mm}$ included ITZs. The cement matrix was modelled by spheres with the diameter $0.35 \mathrm{~mm}$ $\leq d_{c m}<2 \mathrm{~mm}$ without ITZs. ITZs were simulated for the sake of simplicity as contacts between aggregate and cement matrix grains. Thus they had no a physical width in contrast to experiments. Note that simulation of the width of ITZs $(0.010-0.025 \mathrm{~mm})$ requires very small spheres. In 2D calculations, the specimen length $L$ included one row of aggregate and cement matrix particles. The specimen preparation process consisted of 2 stages. Initially aggregate particles and clusters simulating voids were created. Later smaller particles were added until the final specimen was filled in $98.4 \%$ in order to realistically the experimental micro-porosity was measured with $\mu \mathrm{CT}$ as $1.6 \%$ (Section 2.2). After the cement matrix was created, the particles were removed at the place of voids. Next all contact forces due to the particle penetration were deleted. The following five main local material parameters were needed for our discrete 
simulations: $E, v, \mu, C$ and $T$. In addition, the particle radius $R$, particle mass density $\rho$ and damping parameters $\alpha_{d}$ were required.

The following parameters of the cohesion and tensile strength were used in all DEM analyses: cement matrix $\left(E_{c, c m}=15 \mathrm{GPa}, C_{c m}=140 \mathrm{MPa}\right.$ and $\left.T_{c m}=25 \mathrm{MPa}\right)$ and ITZs $\left(E_{c, I T Z}=12 \mathrm{GPa}\right.$, $C_{I T Z}=112 \mathrm{MPa}$ and $\left.T_{I T Z}=20 \mathrm{MPa}\right)$ based on our earlier calculations [3,4,7]. ITZs were obviously the weakest phase. The ratio $E_{c, I T Z} / E_{c, c m}=0.8$ was chosen based on the experiments. The remaining ratios were also assumed as $0.8: C_{I T Z} / C_{c m}=0.8$ and $T_{I T Z} / T_{c m}=0.8$ due to the lack of experimental results. Note that there were no contacts between aggregate grains $\left(d_{a} \geq 2 \mathrm{~mm}\right)$. The remaining parameters were constant for all phases and regions: $v_{c}=0.2$ (Poisson's ratio of grain contact), $\mu=18^{\circ}$ (inter-particle friction angle), $\alpha_{d}=0.08$ (damping parameter) and $\rho=2.6 \mathrm{kG} / \mathrm{m}^{3}$ (mass density). The prescribed damping parameter $\alpha_{d}$ and velocity did not affect the results during bending [7]. In the case of $\alpha_{d}<0.08$, the too excessive kinetic energy was always created during fracture (the tensile numerical test could not be performed without numerical damping due to excessively high velocities of particles (see also [7]). In turn, the effect of the $\alpha_{d}$-value on global results for $\alpha_{d} \geq 0.08$ became insignificant. The 2D concrete specimen under tensile splitting included in total about 20'000 spheres. The material constants were assumed based on the test on concrete not on the cement matrix (due to the lack of the detailed information on properties of ITZs). With the material constants assumed, the uniaxial compressive strength of the $2 \mathrm{D}$ four-phase concrete specimen $\left(15 \times 15 \mathrm{~cm}^{2}\right)$ was about $45 \mathrm{MPa}$ and elastic modulus $E=30 \mathrm{GPa}$ (as in the experiments, Section 2.1).

\section{DEM RESULTS}

The 2D cylindrical concrete specimens of the diameter $D=150 \mathrm{~mm}$ obtained using DEM with two different loading/supporting systems as compared to real specimens were modeled. The deformable strip of the plywood board at the top and bottom of the specimen was created from 240 spheres assembled together with the $50 \%$ lower stiffness than concrete (with this value the same global elastic stiffness was calculated as in the experiment). It had a full contact with the concrete specimen without wall friction. The rigid cylinder of steel at the top and bottom of the specimen was created by a single sphere of the diameter of $20 \mathrm{~mm}$ with the 10-times higher stiffness than concrete. The deformation was induced by prescribing the vertical top displacement in such way that the changes of CMOD were approximately linear in time (as in experiments). CMOD was calculated as a horizontal displacement at the specimen mid-height between mid-points of two regions with the area of $A=5 \times 15 \mathrm{~mm}^{2}$ (based on preliminary calculations). The mid-points were at the distance of $40 \mathrm{~mm}$ as in the experiment (Fig.1). If the calculated CMOD was larger than the assigned CMOD growth $\left(v_{C M O D}=1 \times 10^{-5} \mathrm{~mm} / \mathrm{s}\right)$, the difference was multiplied by the earlier calibrated proportional parameter $n=1.5$ since the inertia of the discrete system was different than this of the testing machine $\left(u_{t+l}=n \times u_{t}\left(\mathrm{CMOD}_{\text {assigned }}{ }^{-}\right.\right.$ $\left.\mathrm{CMOD}_{t}\right)$ ). A too small averaging area $A$ mainly contributed to excessive displacement oscillations. In addition in order to diminish the excessive kinetic energy after the contact breakage between the areas $A$, larger local damping was assumed in calculations $\left(\alpha_{d}^{\text {local }}=0.05\right)$ if the single grain velocity $v_{t}$ was larger than $0.001 \mathrm{~m} / \mathrm{s}$. Next this velocity was reduced in the next step down to $v_{t+1}=\alpha_{d^{\bullet}} v_{t}$. In calculations, the time step $\mathrm{d} t$ was equal to $\mathrm{d} t=10^{-8} \mathrm{~s}$.

Figure 3 presents the DEM results of the strength compared to the experiments. The strength's evolution $\sigma$ versus CMOD and $v$ was satisfactorily reproduced (Figs.3A and 3B). The 
calculated maximum tensile stress was by $2 \%$ too low for the steel loading cylinder and by $10 \%$ too high for the plywood loading board than in experiments. The calculated residual tensile stress was the same for the steel loading cylinder and by $30 \%$ too high for the plywood board. The calculated rate of softening was similar for the steel cylinder and too small for the plywood board. The differences between numerical and experimental results are probably caused by the different stiffness of the plywood board than in experiments and some slight deviations between the calculated and theoretical CMOD evolution.

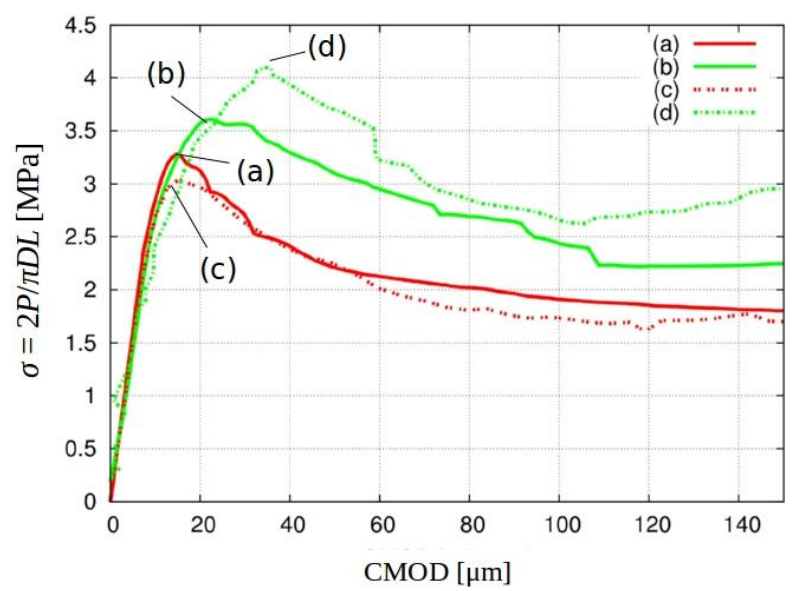

A)

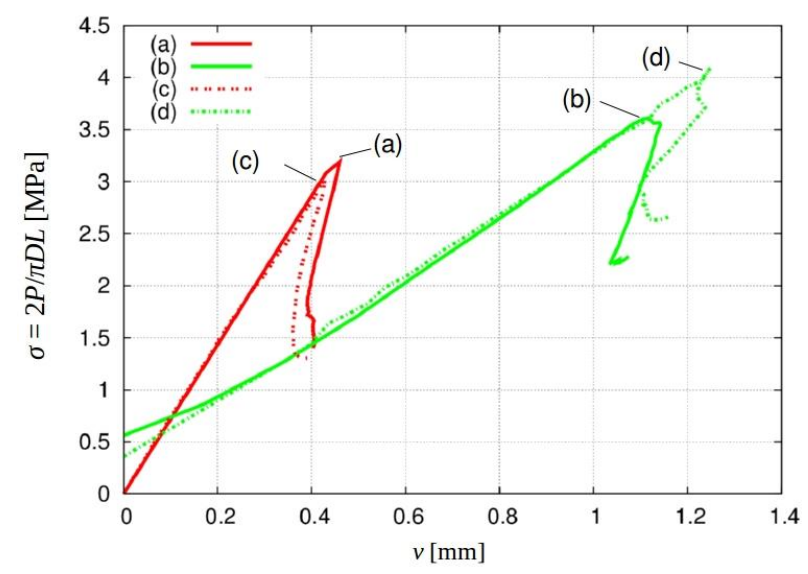

B)

Figure 3: Calculated DEM results against experimental ones: A) strength $\sigma$ against $C M O D, \mathrm{~B}$ ) strength $\sigma$ against top vertical displacement $v$ (B) (curves 'a' and 'b' - experiments, curves 'c' and ' $d$ ' - DEM, red lines - steel loading cylinder, green lines - plywood loading board)in experiment (a) and DEM (b) (continuous lines - experiments, dashed lines - DEM)

The calculated crack patterns were very similar to the experimental ones (Fig.4). In DEM calculations with the plywood strip (Fig.4Bb), the macro-crack followed exactly the same path as in experiments (Fig.4Ba). It also branched into rigid wedges at the same height $(25 \%$ of $D$ from the top and bottom). However, the crack shape in the wedge often followed the other side of the aggregate particle. In DEM calculations with the steel cylinder (Fig.4Ab), the macro-crack was more curved than in experiments (Fig.4Aa) and followed the opposite edges of aggregates in the lower specimen half. The large aggregate grain at the specimen bottom crushed in the experiment in contrast to DEM outcomes (our model has not included grain crushing yet). In DEM simulations, initially several single micro-cracks occurred in the entire specimen. The broken contacts first occurred always in ITZs at corners of aggregate particles wherein tensile forces were the largest (Fig.5Aa). Later micro-cracks started to concentrate in the vertical central zone at the specimen mid-height. Afterwards they connected with each other in the cement matrix by bridging and created a discrete macro crack in the vertical central zone (similarly as in the experiment) (Fig.5Ac). The crack propagated towards the top and bottom of the specimen for $\mathrm{CMOD}=30 \mu \mathrm{m}$. At the top and bottom, it branched by creating a clear wedge under the loading strip. At the test end (CMOD $>150 \mu \mathrm{m})$, the specimen was symmetrically de-fragmented. The macro-cracks were always created by bridging interfacial micro-cracks (Fig.6). 

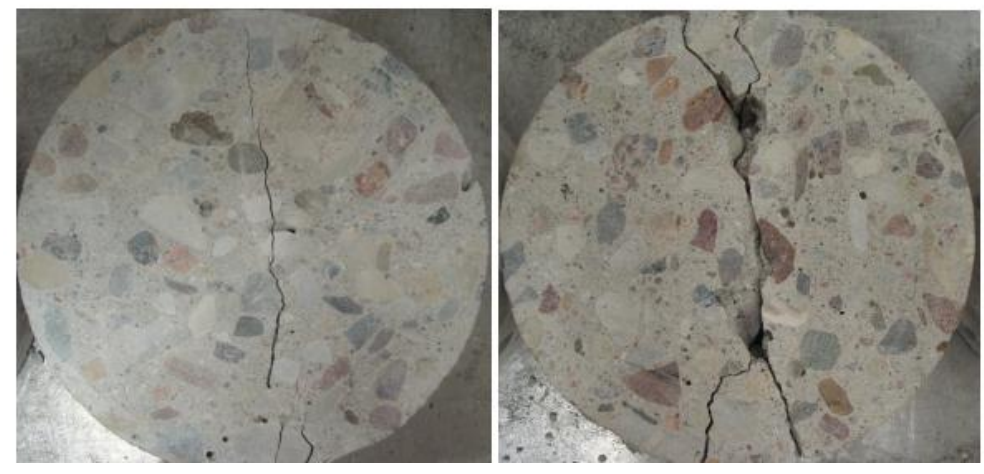

a)

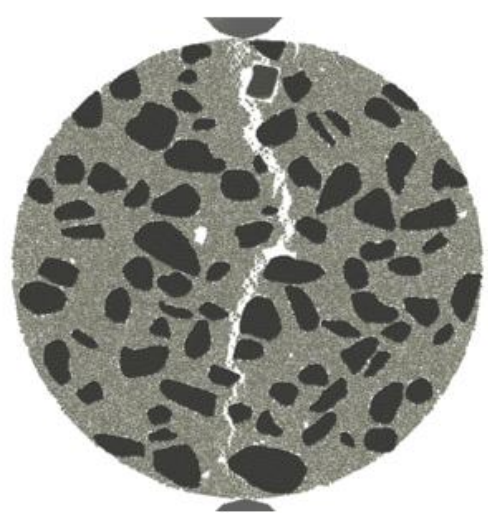

A)

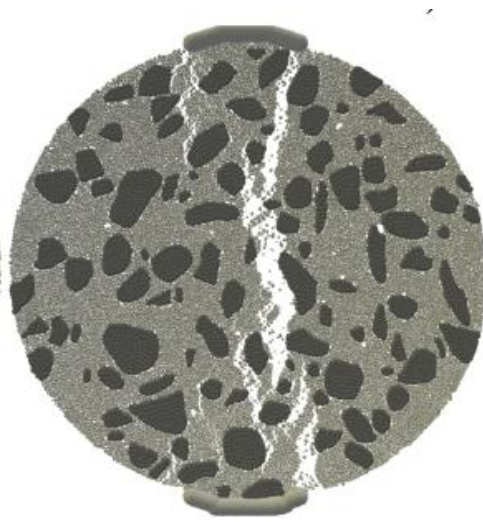

b)

B)

Figure 4: Experimental and calculated fractured specimens at residual state for CMOD $>150 \mu \mathrm{m}$ : a) experimental cracks and b) calculated cracks by DEM for A) steel loading cylinder and B) plywood loading board

Figure 5B presents the evolution of inter-particle normal contact forces. The blue lines indicate tensile and red lines compressive forces. The line thickness is proportional to the force magnitude. The external vertical splitting force was transmitted via a network of normal contact forces which formed force chains. They carried the majority of the loading and transmitted it on the entire system and were the predominant structure of internal forces at micro-scale. Initially large vertical compressive normal contact forces were created in the almost entire specimen (Fig.5Ba). Tensile normal forces occurred in a perpendicular (horizontal) direction. In the boundary regions compression obviously dominated over tension. Before the peak of the vertical force, the compression and tensile forces increased, however some single tensile forces started to break due to the contact damage (Fig.5Bb). After the load peak the horizontal tensile forces started to reduce $(\mathrm{Fig} .5 \mathrm{Bc})$. When a vertical macro-crack occurred in the specimen, the compressive forces concentrated in the specimen mid-region whereas the tensile forces became 
located mainly along the specimen circumference due to the compression of two separated specimen halves (Fig.5Bd).

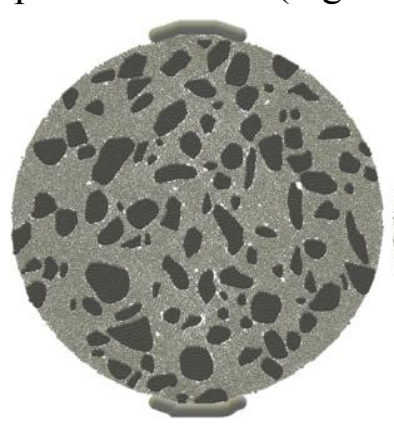

a)

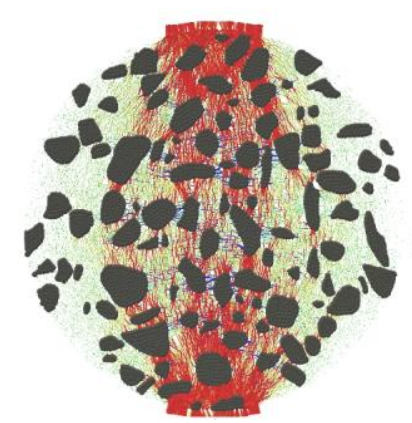

a)

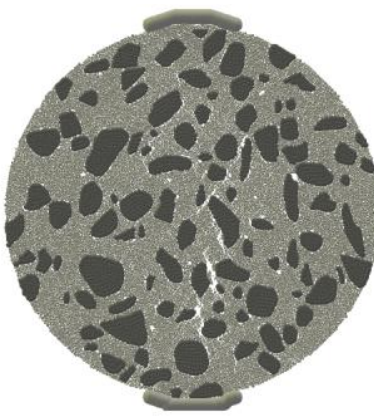

b)

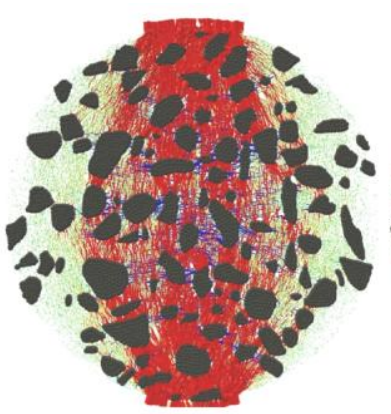

b)

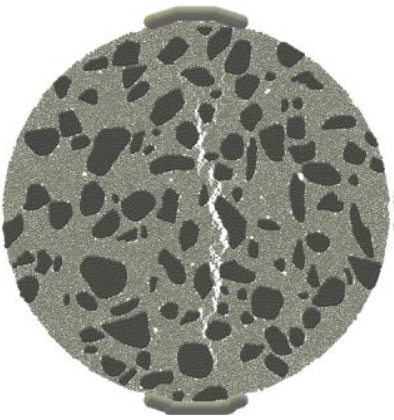

c)

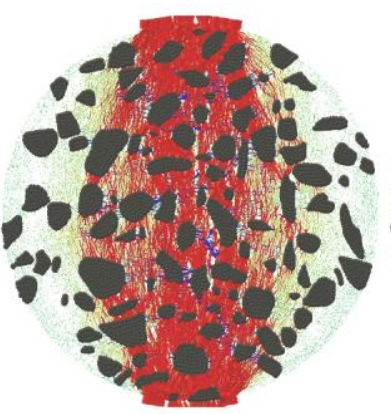

c)



d)

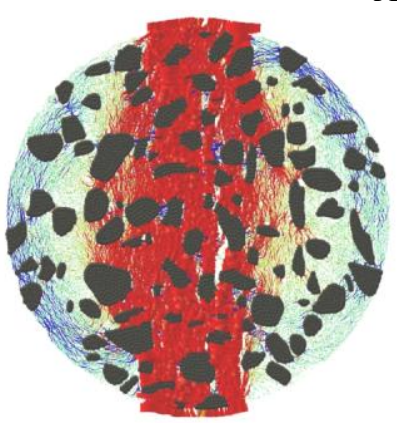

d)

B)

Figure 5: DEM results: A) evolution of cracking in concrete specimen and B) evolution of interparticle tensile (blue lines) and compressive (red lines) normal contact forces for concrete specimen with plywood loading board versus CMOD: a) $\mathrm{CMOD}=10 \mu \mathrm{m}, \mathrm{b}) \mathrm{CMOD}=20 \mu \mathrm{m}, \mathrm{c}$ ) $\mathrm{CMOD}=30 \mu \mathrm{m}$ and $\mathrm{d}$ ) $\mathrm{CMOD}>150 \mu \mathrm{m}$ (green colour denotes small values of internal forces)

The evolution of the total elastic energy was analysed during the continuing deformation process (test with the steel loading cylinder) (Fig.7) without a separation into recoverable and stored terms. For the vertical top displacement $v$ corresponding to the peak load $P_{\max }$ $(\mathrm{CMOD}=20 \mu \mathrm{m}, v=0.46 \mathrm{~mm}$ ) (Fig.7A), the elastic internal energ $y$ was equal to $75 \%$ (normal energy - 52\%, tangential energy - $23 \%$ ), plastic dissipation was equal to $1 \%$, energy of debonded contacts was equal to $2 \%$, kinetic energy was equal to $0.5 \%$ and numerical damping was equal to $21.5 \%$ of the total energy. At the failure $(v=0.43 \mathrm{~mm}, \mathrm{CMOD}=150 \mu \mathrm{m})$, the elastic internal energy was $42 \%$, plastic dissipation was $2.5 \%$, contact breakage energy was equal to $5 \%$, kinetic energy was $0.5 \%$ and numerical damping was $50 \%$ of the total energy. Due to the snap-back instability, the total internal energy reduced by $15 \%$, the elastic normal internal energy reduced by $60 \%$ and the elastic tangential internal energy reduced by $20 \%$ (Fig.23B). In turn, the plastic dissipation, numerical damping and elastic energy from removed cohesive contacts increased by the factor 2.5, 2 and 2.5, respectively. 


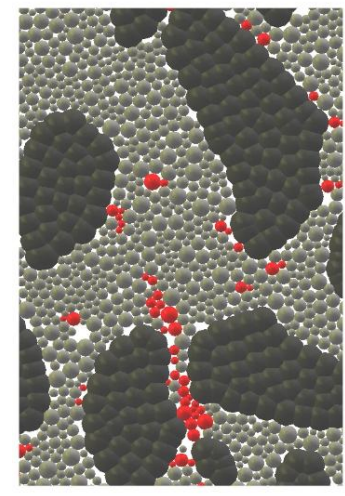

a)

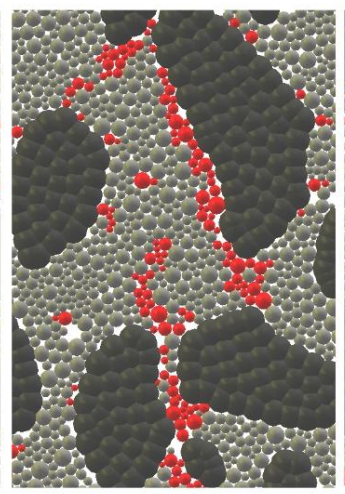

b)

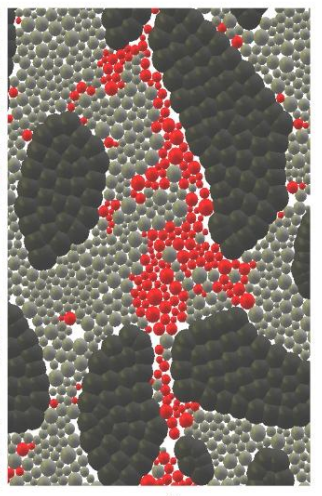

c)

Figure 6: Mechanism of macro-crack creation in concrete specimen by bridging interfacial zones for plywood loading board: a) $\mathrm{CMOD}=10 \mu \mathrm{m}$, b) $\mathrm{CMOD}=15 \mu \mathrm{m}$ and c) $\mathrm{CMOD}=20 \mu \mathrm{m}$ (spheres in red indicate broken contacts and dark grey corresponds to aggregate)

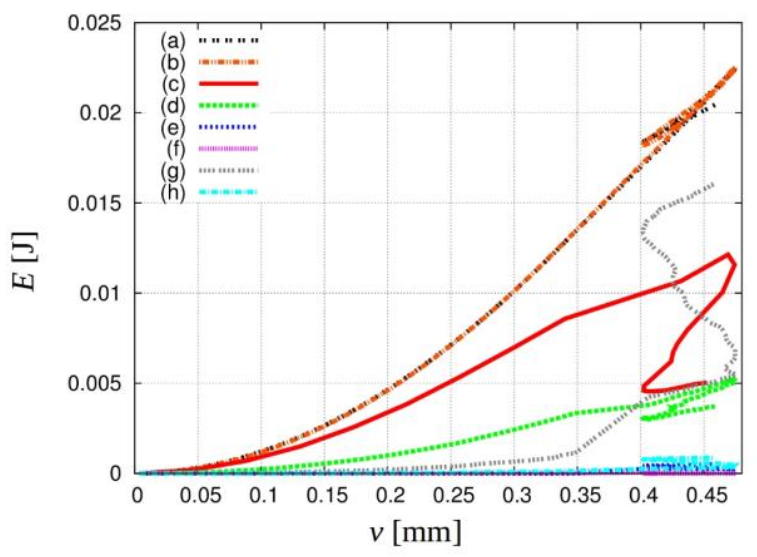

A)

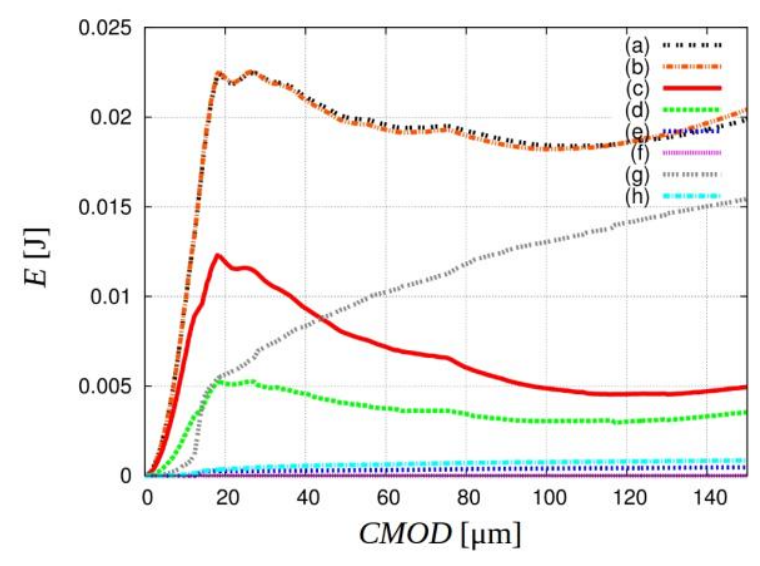

B)

Figure 7: Energy evolution in 2D concrete specimen with steel loading cylinder versus vertical top displacement $v(\mathrm{~A})$ and CMOD (b) using DEM: a) external work, b) internal work, c) normal elastic energy, d) tangential elastic energy, e) plastic dissipation, f) kinetic energy, g) numerical damping and $\mathrm{h}$ ) energy of debonded particles

In order to improve the numerical results with respect to the experiments, the DEM model will be enhanced by aggregate crushing, width of ITZs and 3D analyses. The experiments and DEM calculations will be continued for the different specimen diameters $(D=5-30 \mathrm{~cm})$.

\section{ACKNOWLEDGEMENTS}

The research work has been carried out within the project "Experimental and numerical analysis of coupled deterministic-statistical size effect in brittle materials" financed by the Polish National Science Centre NCN (UMO-2013/09/B/ST8/03598). 


\section{CONCLUSIONS}

- $\quad$ The type of the loading strip significantly affected the concrete behaviour. The strength was smaller by $10 \%$, CMOD corresponding to the strength was smaller by $20 \%$ and vertical displacement corresponding to the strength was smaller by $300 \%$ when a line loading contact was prescribed at the top. In experiments, a snap-back phenomenon occurred. A clear compressive wedge took place when plywood loading/supporting boards was used that caused the strength's increase.

- $\quad$ The macro-crack was curved along the specimen height and length due to a stochastic distribution of aggregates. It was initiated in the central region at the mid-height. Initially micro-cracks always occurred in ITZs and then propagated through a bridging mechanism. Several aggregates crushed during experiments. The width of ITZs was about $10-25 \mu \mathrm{m}$.

- $\quad$ DEM proved its capability to model concrete fracture in detail by taking the snap-back instability into account. The agreement of calculated stress-displacement results and crack shapes with experimental ones was satisfactory. A decrease of the strength of ITZs and minimum particle diameter in the mortar caused the reduction of both the splitting tensile strength and material brittleness.

- The external vertical splitting force was transmitted via a network of normal contact forces which formed clear force chains. Some compressive forces appeared also along the macro-crack edge due to aggregate inter-locking.

- Due to the snap-back instability, the total internal energy reduced by $15 \%$, the elastic normal internal energy reduced by $60 \%$ and the elastic tangential internal energy reduced by $20 \%$. The plastic dissipation, numerical damping and elastic energy from removed cohesive contacts increased by the factor $2.5,2$ and 2.5 , respectively.

\section{REFERENCES}

[1] Tejchman, J. and Bobiński, J. Continuous and discontinuous modeling of fracture in concrete using FEM. Springer, Berlin-Heidelberg (eds. W. Wu and R. I. Borja), 2013.

[2] Skarżyński L. and Tejchman J. Experimental investigations of fracture process in concrete by means of x-ray micro-computed tomography. Strain (2016) 52:26-45.

[3] Skarzynski, L. Nitka, M. and Tejchman, J. Modelling of concrete fracture at aggregate level using FEM and DEM based on X-ray CT images of internal structure. Engineering Fracture Mechanics (2015) 147:13-35.

[4] Suchorzewski, J. Tejchman, J. and Nitka M. Discrete element method simulations of fracture in concrete under uniaxial compression based on its real internal structure. International Journal of Damage Mechanics (2017) 3:. DOI: $10.1177 / 1056789517690915$.

[5] ASTM, Standard Test Method for Splitting Tensile Strength of Cylindrical Concrete Specimens, C496M-04, 2004.

[6] Kozicki, J. and Donze, F.V. A new open-source software developer for numerical simulations using discrete modeling methods. Computer Methods in Applied Mechanics and Engineering (2008) 197:4429-4443.

[7] Nitka, M. Tejchman. J. Modelling of concrete behaviour in uniaxial compression and tension with DEM. Granular Matter (2015) 17(1): 145-164. 